\title{
Long term outcomes of inversion ankle injuries
}

\section{A Anandacoomarasamy, L Barnsley}

Br J Sports Med 2005;39:e14 (http://www.bjsportmed.com/cgi/content/full/39/3/e14). doi: 10.1136/bjsm.2004.011676

See end of article for authors' affiliations .....................

Correspondence to: Associate

Professor Barnsley, Department of Rheumatology, Concord Hospital, Hospital Road, Concord, NSW 2139, Australia; les.barnsley@ email.cs.nsw.gov.au

Accepted 16 March 2004
Background: Ankle sprains are common sporting injuries generally believed to be benign and self limiting. However, some studies report a significant proportion of patients with ankle sprains having persistent symptoms for months or even years.

Aims: To determine the proportion of patients presenting to an Australian sports medicine clinic who had long term symptoms after a sports related inversion ankle sprain.

Methods: Consecutive patients referred to the NSW Institute of Sports Medicine from August 1999 to August 2002 with inversion ankle sprain were included. Exclusion criteria were fracture, ankle surgery, or concurrent lower limb problems. A control group, matched for age and sex, was recruited from patients attending the clinic for upper limb injuries in the same time period. Current ankle symptoms, ankle related disability, and current health status were ascertained through a structured telephone interview.

Results: Nineteen patients and matched controls were recruited and interviewed. The mean age in the ankle group was 20 (range 13-28). Twelve patients (63\%) were male. Average follow up was 29 months. Only five (26\%) ankle injured patients had recovered fully, with no pain, swelling, giving way, or weakness at follow up. None of the control group reported these symptoms $(p<0.0001)$. Assessments of quality of life using short form-36 questionnaires (SF36) revealed a difference in the general health subscale between the two groups, favouring the control arm $(p<0.05)$. There were no significant differences in the other SF36 subscales between the two groups.

Conclusion: Most patients who sustained an inversion ankle injury at sport and who were subsequently referred to a sports medicine clinic had persistent symptoms for at least two years after their injury. This reinforces the importance of prevention and early effective treatment.
A cute ankle injuries are one of the most common problems seen in sports medicine. ${ }^{1}$ In some series, it is the most common injury seen. ${ }^{2}$ Ankle sprains can account for up to $45 \%$ of sports related injuries in high risk sports such as basketball. ${ }^{3}$ Most ankle sprains involve the lateral ligament complex of the ankle joint. ${ }^{4}$ The incidence of ankle sprains has been estimated at 1 per 10000 persons per day. ${ }^{3}$ These injuries are widely perceived to be benign and self limiting, responding well to conservative management. ${ }^{5}$ However, a number of studies have shown that residual symptoms months to years after the injury are not uncommon. The consequences include mechanical instability, intermittent swelling and stiffness, and accumulation of cartilage damage leading to degenerative changes. ${ }^{4}$

Previous studies have examined the consequences of ankle sprains in different population settings. Braun, ${ }^{6}$ in general practice, reports residual symptoms in $72 \%$ of patients 618 months after an ankle sprain. Konradsen et $a l^{7}$ found chronic complaints of pain, swelling, or recurrent sprains at a seven year follow up in $32 \%$ of patients who had been seen in their emergency department. This group included nonsporting injuries. A study among young military recruits examined the outcomes of all ankle injuries at six weeks and six months, and noted residual symptoms in $40 \%{ }^{2}$ Among Chinese athletes, persistent symptoms were found in up to $30 \%$ of participants. However, this study included all types of ankle injuries, and follow up time was not reported. $^{8}$

This study aimed to evaluate the long term outcomes of inversion ankle injuries sustained during sport. This group was chosen as these subjects are generally motivated and fit, and would be the most likely group to succeed with treatment regimens. The secondary aims were to assess potential predictors of outcome, such as age, sex, type of sport, previous injury, and recurrence of injury.

\section{METHODS}

This study was conducted at the NSW Institute of Sports Medicine (NSW IOSM) at Concord Hospital, Sydney. Concord Hospital is a tertiary referral hospital in metropolitan Sydney. The NSW IOSM receives referrals from the emergency department at Concord Hospital, general practitioners, and physiotherapists. The patients range from elite level sportsmen and women to recreational athletes. The institute also sees children with sporting injuries.

The study was designed as a retrospective case-control evaluation. Consecutive patients with acute ankle inversion injuries sustained between August 1999 and August 2002 were eligible for inclusion.

Patients had to meet the following inclusion and exclusion criteria for entry into the study. There was no age cut off for entry. Inclusion criteria were: $(a)$ inversion ankle injury; (b) injury sustained during sporting activity. Exclusion criteria were: (a) other lower limb injury; $(b)$ concurrent fracture with or without dislocation or with or without complete rupture; (c) surgery for ankle injury. The Human Research Ethics Committee at Concord Hospital granted ethics approval for the study.

All patients had undergone plain $x$ ray imaging at initial assessment. They were managed acutely with RICE (rest, ice, compression, elevation) and then with early, controlled mobilisation.

The control group was recruited from patients attending the institute for upper limb injuries over the same time period with no history of lower limb injuries. These patients were matched for age, sex, and, where possible, sport.

All potential subjects and controls were contacted by mail with an information sheet and covering letter indicating that they would be telephoned. The letter included the option of not participating. Telephone calls were placed to each potential subject and control. If they agreed to participate 


\begin{tabular}{l} 
Table 1 Variables assessed with the ankle \\
specific questionnaire \\
\hline Residual pain \\
Pain score \\
Site of pain \\
Pain with rest or activity \\
- Use of analgesia/ anti-inflammatory drugs \\
- Ongoing treatment (for example, physiotherapy or \\
toping) \\
Swelling \\
- Giving way \\
- Recurness \\
Return to sport, time to return to sport \\
Sport played at time of injury \\
Frequency of sport at time of injury \\
- Ooss of time from work or study at injury \\
- Participation in a physiotherapy programme \\
\hline
\end{tabular}

and provided consent, two questionnaires were administered by structured interview. Each interview took about 30 minutes.

The first questionnaire was ankle specific, and the second was the short form-36 quality of life questionnaire (SF36). The ankle specific questionnaire was developed for the purpose of this study. It assessed residual pain, swelling, weakness, instability, ongoing treatment, recurrence of injury, prior injury, return to sport, return to work, and adherence to physiotherapy among other things. Table 1 presents details of the questionnaire.

At the time of initiation of the study, ankle specific questionnaires did not exist in the literature. Subsequent to the start of this study, a sports ankle rating system was proposed as a validated tool. ${ }^{9}$ The variables we considered, together with the SF36, were similar to those assessed in the questionnaire component of the sports ankle rating system.

The primary outcome measure was the proportion of patients still with symptoms in each group. The proportions were compared using Fisher's exact test. The SF36 scales were compared using Student's two sample $t$ test for samples with unequal variances.

\section{RESULTS}

Forty seven patients presented with acute ankle inversion injuries to the NSW IOSM between August 1999 and August 2002. Of these, 21 met eligibility criteria. Nineteen of these patients were contacted and agreed to participate (response rate $90 \%$ ). The remaining two patients were overseas. Ten subjects had injured their right ankle, and the remaining nine had injured their left ankle.

A total of 103 patients had presented to the NSW IOSM with upper limb injuries between August 1999 and August 2002. After screening, 68 met eligibility criteria. Consecutive patients were then matched for age, sex, and, where possible sport. Of 21 matched patients who were eligible to

Table 2 Baseline characteristics of the two groups

\begin{tabular}{lll}
\hline & Ankle & Control \\
\hline Mean age (years) & 20 & 22 \\
Range of age (years) & $13-28$ & $10-42$ \\
Mean follow up (months) & 29 & 28 \\
Range of follow up (months) & $12.6-48$ & $12.5-46$ \\
Females & 7 & 6 \\
Males & 12 & 13 \\
\hline
\end{tabular}

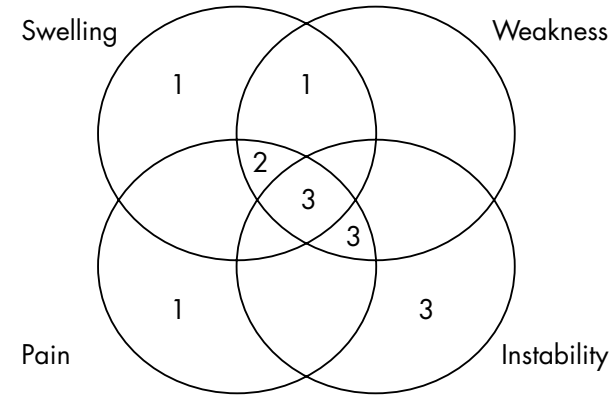

Figure 1 Combinations of symptoms observed in the ankle injured group.

participate, there were 19 respondents. One patient was overseas, and the other declined to participate because of work commitments. Table 2 presents the characteristics of the two groups. Four of the seven women and nine of the 12 men in the ankle group also matched for sport with the control group (total proportion matched for sport $\sim 70 \%$ ).

On the basis of the ankle specific questionnaire, 14 patients (74\%) in the ankle group had at least one symptom at the time of follow up. Nine patients $(47 \%)$ had pain, nine had perceived instability, nine had weakness, and seven (37\%) had swelling. Nine patients $(47 \%)$ had more than one symptom at follow up. Figure 1 illustrates the combination of symptoms reported.

In contrast, none of the patients in the control group had any of the symptoms of ankle pain, swelling, instability, or weakness. The difference between the two groups of having at least one symptom was highly significant $(\mathrm{p}<0.0001)$.

\section{Sports}

Most patients in the ankle group (12 of 19) were involved in a football code. Ten of the 19 patients in the control group were involved in a football code. Table 3 lists the sports in which the subjects injured their ankles.

Sixteen patients with ankle injuries returned to their original sport. The remaining three were persistently impaired by their ankle injury. Two changed to other sports because of their ankle symptoms, and one did not return to sport. The mean time to return to sports in the 18 patients who returned to sport was 12.9 weeks (range 2 weeks to 18 months). Two patients were delayed by non-ankle related reasons: one was pregnant, and the other got married.

\section{Work/study}

Time to return to work after injury ranged from less than one week to two months. No-one encountered ongoing loss of work or study time on the basis of his or her ankle injury.

\section{Prior injury and recurrence}

Five of the 19 subjects had a recurrent ankle injury as their index event. Of these, two had a further injury during follow up. A total of eight $(42 \%)$ of the 19 patients had recurrent

Table 3 Details of sport type in the ankle group

\begin{tabular}{ll}
\hline Football code & Other \\
\hline Rugby (3) & Aerobics \\
Soccer (7) & Basketball \\
Touch football (2) & Cycling \\
& Dance sports \\
& Karate (2) \\
& Tennis \\
\hline
\end{tabular}


What is already known on this topic

- Inversion ankle injuries are one of the most common sporting injuries.

- Residual symptoms of pain, swelling, weakness, and instability have been reported in certain populations.

injuries during follow up, with seven having persistent symptoms.

\section{Physiotherapy}

Sixteen patients underwent a supervised physiotherapy programme. Of these, 14 patients were treated at the NSW IOSM, and the other two sought treatment elsewhere. Three did not attend physiotherapy.

\section{Ongoing treatment}

Six patients regularly used an external brace or applied adhesive taping for sports. Of these, three patients had recurrent injuries.

\section{Quality of life measures (SF36)}

A significant difference was noted in the general health scale between the ankle and control group, favouring the latter $(p<0.05)$. No significant differences in the other SF36 scales (physical functioning, role-physical, bodily pain, vitality, social functioning, role-emotional and mental health) were seen between the two groups.

\section{DISCUSSION}

Our results reveal a remarkably high rate of persisting symptoms after ankle inversion injury. Nearly three quarters $(74 \%)$ had at least one symptom when assessed $1.5-4$ years after their injury. It was interesting that, despite the persistence of residual symptoms, most patients returned to sport. No relation was noted between age, sex, and type of sport with long term outcomes. However, most of the patients (seven of eight) with recurrent injuries had persistent symptoms. We could not identify a relation between prior injury and recurrence. As the numbers were small, no subgroup analysis was attempted on the other data.

This is the first study, to our knowledge, that was specifically designed to assess the outcomes of ankle inversion injuries in sport. Previous studies have assessed the outcomes of ankle sprains seen in general practice and the emergency department. These studies have included ankle sprains sustained during non-sporting injuries. The study by Yeung et $\mathrm{al}^{8}$ examined the effects of ankle injuries in athletes. This study was a cross sectional survey of athletes with prior ankle sprains and did not stipulate whether these were inversion injuries or complete ligamentous ruptures or fractures. Even so, our study reflects results from previous studies pertaining to ankle sprains, albeit with different populations and in different circumstances, that a significant number of patients are troubled by persistent symptoms after their ankle injury.

The response rate for this study was excellent, and we were able to follow up almost all the eligible patients. This eliminates any potential bias from non-respondents. The treatment strategies used at NSW IOSM are similar to those generally accepted in the literature, so it is unlikely that idiosyncratic treatments at our institution are responsible for these results. Patients were carefully investigated with additional imaging, looking for other injuries such as talar dome fractures when they had atypical or particularly troublesome symptoms. We also did not differentiate
What this study adds

- This is the first study to assess long term outcomes in inversion ankle injuries secondary to sport.

- $74 \%$ of the patients had persisting symptoms 1.5-4 years after their injury.

- There is a need for more effective treatment and preventive strategies to reduce the high incidence of residual symptoms.

between grade 1 and grade 2 injuries as this grading system has not been shown to relate to prognosis. ${ }^{10}$

Our study has some limitations. Firstly, it is retrospective and the numbers are small. Owing to logistical difficulties such as patients moving interstate or living in rural NSW, the follow up was based on a telephone questionnaire and did not include clinical examination. Functional testing would have provided more objective information. Sports physicians, rheumatologists, and orthopaedic surgeons treat the patients at the NSW IOSM, and consequently there may be subtle differences in management styles. Not everyone attended physiotherapy at the NSW IOSM, and treatment was therefore not standardised. There were also differences in ongoing treatment with regard to taping and bracing. Predisposing conditions to ankle injuries were not specifically sought-for example, hypermobility syndrome. Finally, we did not assess whether patients returned to their previous level of sport-for example, recreational compared with competitive.

Given that the ankle and control groups were largely matched for age, sex, and sport, it raises the question of whether some people are more prone to ankle injuries because of biomechanical and other factors. Consequently, their recovery may be more prolonged, and they may require more aggressive and targeted treatment. Further studies to evaluate the possible identification and treatment of high risk sportspeople are required.

This study reinforces the notion that ankle sprains are not just simple injuries. An appreciable number of patients are impaired by persistent ankle symptoms, which may lead to long term disability and instability. It also highlights the need for more effective management strategies, further research, and ongoing education on prevention during training sessions in the various sports.

\section{ACKNOWLEDGEMENTS}

We acknowledge the invaluable contribution by Sandra Campbell, Secretary NSW IOSM, for her help in accessing the data required. We also thank all the patients for participating in the study.

\section{Authors' affiliations}

A Anandacoomarasamy, L Barnsley, Department of Rheumatology, Concord Hospital, Concord, NSW, Australia

Competing interests: none declared

\section{REFERENCES}

1 Garrick JG, Requa RK. The epidemiology of foot and ankle injuries in sports. Clin Podiatr Med Surg 1989;6:629-37.

2 Gerber JP, Williams GN, Scoville CR, et al. Persistent disability associated with ankle sprains: a prospective examination of an athletic population. Foot Ankle Int 1998; 19:653-60

3 Trevino SG, Davis P, Hecht PJ. Management of acute and chronic lateral ligament injuries of the ankle. Orthop Clin North Am 1994;25:1-16.

4 Struijs P, Kerkhoffs G. Ankle sprain. Clin Evid 2002;(7):945-953.

5 Groff GD. Ankle sprain. In: Klippel J, Dieppe P, eds. Rheumatology. London: Mosby, 1998:14.10-14.13. 
6 Braun BL. Effects of ankle sprain in a general clinic population 6 to 18 months after medical evaluation. Arch Fam Med 1999;8:143-8.

7 Konradsen L, Bech L, Ehrenbjerg M, et al. Seven years follow-up after ankle inversion trauma. Scand J Med Sci Sports 2002; 12:129-35.

8 Yeung MS, Chan KM, So $\mathrm{CH}$, et al. An epidemiological survey on ankle sprain. Br J Sports Med 1994;28:112-16.

9 Williams GN, Molloy JM, DeBerardino TM, et al. Evaluation of the sports ankle rating system in young, athletic individuals with acute lateral ankle sprains. Foot Ankle Int 2003;24:274-82.

10 Cross KM, Worrell TW, Leslie JE, et al. The relationship between self-reported and clinical measures and the number of days to return to sport following acute lateral ankle sprains. J Orthop Sports Phys Ther 2002;32:16-23.

\section{COMMENTARY}

As an orthopaedic surgeon specialising in foot and ankle surgery, I found this paper interesting and stimulating. All too often lateral ankle injuries are viewed as benign by the medical (orthopaedic, rheumatological, sports medicine, general practice) and general populations. I see many patients with ankle sprain that does not heal and believe that a considerable proportion of these injuries result in persistent disability, particularly in the athlete. This paper clearly shows that many patients still experience considerable long term symptoms after this type of injury. The authors acknowledge the limitations of the study (retrospective, small patient numbers, and telephone follow up); however, this study does lead the way for further studies on the conclusions drawn. I believe it will stimulate the clinician to regard these injuries as a potential cause of long term disability, particularly in the athletic population.

L Grujic

Royal North Shore Hospital, Foot and Ankle Surgeon, Level 2, 445 Victoria Avenue, Chatswood, NSW 2067, Australia; talus@optushome.com.au 